\title{
One Pot Three Component Diastereoselective Synthesis of Tricyclic Furoquinolones and Furocoumarins
}

\author{
Reshma Naik ${ }^{1}$ Manohar V Kulkarni ${ }^{1 *}$, Anil Kumar² and T. N. Guru Row ${ }^{3}$ \\ ${ }^{1 *}$ Department of Chemistry Karnatak University, Dharwad 580003, India \\ ${ }^{2}$ Department of Physics, M.S. Ramaiah Institute of Technology, MSRIT post, MSR Nagar, \\ Bangalore-560054 \\ ${ }^{3}$ Solid State and Structural Chemistry Unit, Indian Institute of Science, Bangalore 560 012, India \\ E-mail: manohar274@gmail.com
}

\begin{abstract}
A new synthetic strategy for the construction of dihydrofuro coumarins and quinolones has been developed by a three component reaction. Formation of 2,3-dihydrofuran ring and the trans orientation is supported by ${ }^{1} \mathrm{H}-\mathrm{NMR}$ and confirmed single X-ray diffraction studies.
\end{abstract}

Keywords: One-pot multicomponent, furo-coumarin, furo-quinoline, X-ray, diastereoselective synthesis, 4-hydroxy-coumarin and carbostyril.

\section{Introduction}

Multicomponent reactions (MCR) have played an important role in modern organic synthesis since they provide a short route to moderately complex molecular assemblies in a stereo-selective fashion [1]. Multi component strategies have been developed for asymmetric synthesis [2], natural product synthesis [3] and diversity oriented organic synthesis [4]. Three component reactions have been employed for the synthesis of a variety of heterocycles [5]. Coumarins are a group of naturally occurring lactones, the derivatives of which have been obtained by employing the multicomponent reaction methodology. 3Bromocoumarins [6], 3-amino coumarins [7], and tricyclic pyrimidine fused coumarins [8] have been synthesized by a three component reaction strategy from salicylaldehydes. 4-hydroxy coumarins have been used in the construction of pyran and furan fused tricyclic systems [9-11]. Ugi-4CR reactions involving coumarin 3-carboxylic acid were employed for the construction of amides with lipophilic spacers [12] and chromeno [3,4-c] pyrroles [13].

Quinoline and dihydroquinoline fused tetracyclic coumarins have been generated by a three component reaction of 4-hydroxy coumarins, aromatic amines and aldehydes [14]. Biginelli multi component reaction strategy using coumarin-3- $\beta$-ketoester, aromatic aldehydes and urea/thiourea has resulted in fluorescent 4-3' coumarinyl dihydropyrimidinones and pyrimidinthiones [15]. An interesting non-Ugi $4 \mathrm{CR}$ has been reported on coumarin $3-\beta$-ketoester with chlorobenzaldehydes, phenylhydrazine and malononitrile leading to a one pot synthesis of coumarin linked dihydropyrano [2,3-c] pyrazoles [16]. Recently, Lanthanum triflate catalyzed diastereoselective synthesis of fluorescent indolyl malonamides has been reported by using a three component reaction between coumarin 3-carboxylates, indoles and aromatic amines [17].

Dihydro furo [3,2-c]coumarin and corresponding quinolone skeleton has been a part of many naturally occurring compounds (Figure 1) [18-22]. It is pertinent to mention that dihydro furoquinolones have been found to exhibit broad spectrum of anti-fungal activity [23-24]. Corresponding furocoumarins with trans orientation of the groups at C-2 and C-3 positions in the furan ring exhibited anti-mitotic activity which synergistically enhanced the action of paclitaxel [25]. Naturally occurring 5-methyl furocoumarins have been found to be inhibitors of Plasmodium falciparum [26].

The tricyclic dihydroquinolones have been synthesized by photochemical transformations [27], $2+3$ cycloaddition [28], Silver (I) promoted oxidative addition [29] and stepwise approach [30]. The corresponding oxygen analogues have been synthesized from 4-O-alkylated coumarins [31], Rhodium (II) 


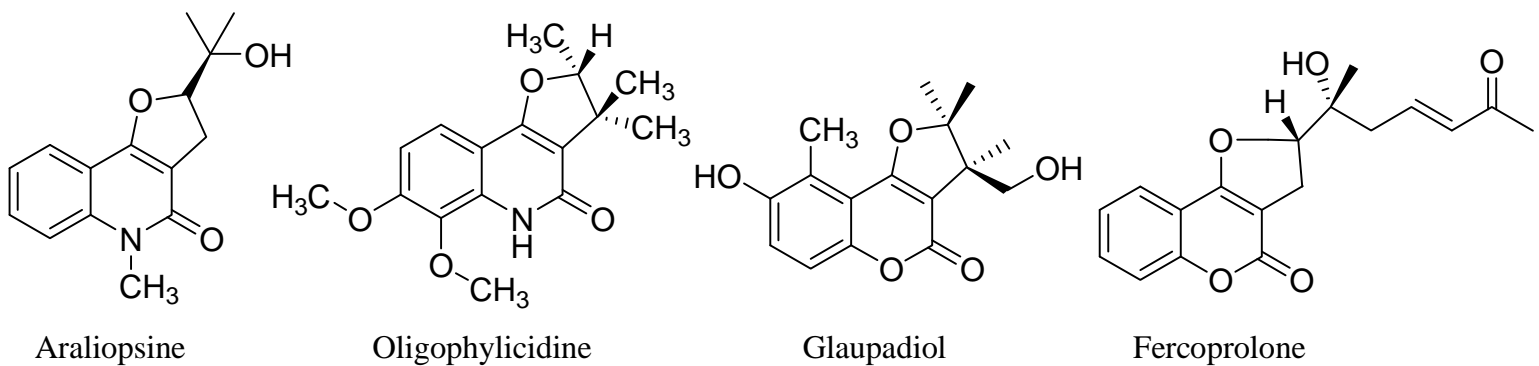

Figure 1. Naturally occurring dihydro [3,2-c] furo quinolones and coumarins

catalysed reaction of cyclic diazodicarbonyl compounds [32], tandem reactions of 4-hydroxycoumarins [33] and other routes [34]. In view of the hitherto mentioned variety of synthetic approaches for the two isosteric tricyclic skeletons, it is quite challenging to evolve a common synthetic strategy through which the two skeletons can be generated in a single step. We have shown that 2,3-dihydro benzofurans linked to C-4 position of coumarin moiety can be generated by the room temperature intramolecular aldol reaction of ortho carbonylated 4-aryloxymethyl coumarins [35] or the corresponding imines [36]. In continuation of our earlier work, design of a three component reaction was envisaged (Scheme 1). The ambident nucleophilicity of 4-hydroxy coumarin and 4-hydroxy carbostyril was effectively used in-situ, in a cascaded manner in its reaction with aromatic aldehydes and 4-bromomethyl coumarins in presence of pyridine and triethylamine.

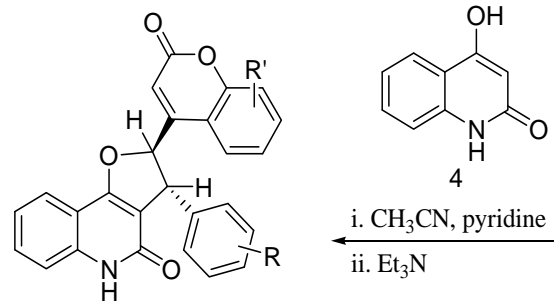<smiles>[R]c1ccc2oc(=O)cc(CBr)c2c1</smiles>

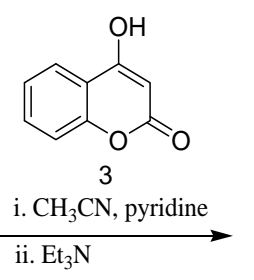

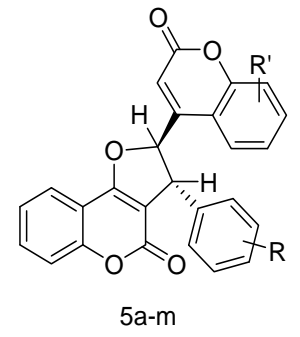

$5 a-m$ $5 n-t$

Scheme 1. Synthesis of furo[3,2-c]chromen-4-one/ quinolin-4(5H)-ones by one-pot reaction.

\section{Results and Discussions}

The required substituted 4-bromomethylcoumarins [37] 1 were prepared by the Pechmann cyclization of substituted phenols with 4-bromoethylacetoacetate [38] using sulfuric acid as the condensing agent.

The three component reaction between 4-bromomethyl coumarins $\mathbf{1}$ and benzaldehyde $\mathbf{2}(\mathrm{R}=\mathrm{H})$ and 4-hydroxy coumarin $\mathbf{3}$ in presence of pyridine using chloroform as solvent at room temperature resulted in the formation of a white crystalline solid corresponding to the 4-coumarinomethyl pyridinium hydrobromide. Employing reflux conditions and using acetonitrile as solvent also did not bring about any change as the expected Knoevenagel condensation between 4-hydroxy coumarin $\mathbf{3}$ and benzaldehyde 2. Hence it was thought of using another base in conjunction with pyridine allowing sufficient time for the two reactive species to be generated. The three reactants viz. 4-bromomethyl coumarin $\mathbf{1}$, aromatic aldehyde $\mathbf{2}$ and 4-hydroxy coumarin 3 were refluxed in acetonitrile for $2 \mathrm{~h}$, using three equivalents of pyridine followed by the addition of one equivalent of triethyl amine. The reaction conditions ensured the formation of pyridinium bromide and a Knoevenagel product from Benzaldehyde 2 and 4-hydroxy coumarin 3. Excess of base in this reaction was found to be necessary for the in-situ generation of the ylide stabilized carbanion at the allylic position of coumarin. Refluxing was continued overnight, the usual work up and purification afforded the tricyclic furanocoumarins 5 .

In a typical case, compound $5 \mathrm{k}$, three proton singlets at 2.24 and $3.80 \mathrm{ppm}$ indicated methyl and methoxy group respectively. Protons in the dihydrofuran ring appeared as doublets at 6.17 and 4.44 $\operatorname{ppm}(\mathrm{J}=4.8 \mathrm{~Hz})$ which are assigned to $\mathrm{C} 2-\mathrm{H}$ and $\mathrm{C} 3-\mathrm{H}$ respectively. Assignment of the downfield signal to the $\mathrm{C} 2-\mathrm{H}$ is also confirmed by its allylic relationship with $\mathrm{C} 3-\mathrm{H}$ of coumarin, which is reflected 
in compound $5 \mathrm{~d}$ in which the $\mathrm{C} 2-\mathrm{H}$ at $6.59 \mathrm{ppm}$ appears as doublet of doublet $(\mathrm{J}=0.8,5.6 \mathrm{~Hz})$, the $\mathrm{C} 3-\mathrm{H}$ of coumarin appears at $6.35 \mathrm{ppm}$ as doublet $(\mathrm{J}=0.8 \mathrm{~Hz})$ and the $\mathrm{C} 3-\mathrm{H}$ of dihydrofuran is observed at $4.53 \mathrm{ppm}$ as doublet $(\mathrm{J}=5.6 \mathrm{~Hz})$. Observed $\mathrm{J}$ values are in conformity with reported $3 \mathrm{JH}-\mathrm{H}$ values for the trans- 2,3 dihydrofurans [29]. X-ray structure for 5k (CCDC 898526) which is solvated with acetonitrile provides a proof for the proposed stereochemistry (Figure 2). Crystal determination and refinement data of compound $5 \mathrm{k}$ are provided in Table 1. Lactone carbonyl oxygen of coumarin shows the intermolecular association with the $\mathrm{CH} 3$ group and $\mathrm{C} 8-\mathrm{H}$ is associated with nitrogen atom of acetonitrile.

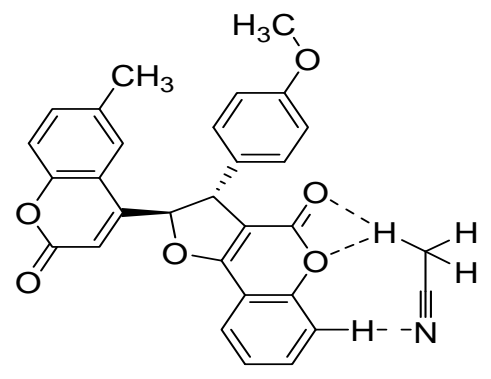

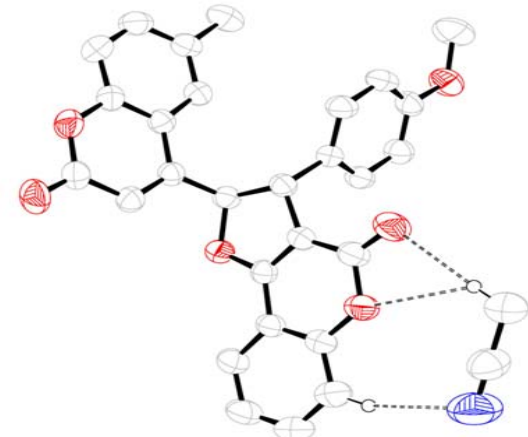

Figure 2. ORTEP diagram of $5 \mathrm{k}$ as acetonitrile solvate

Table 1. Crystal and experimental data of compound $5 \mathrm{k}$

\begin{tabular}{|c|c|}
\hline \multicolumn{2}{|l|}{ Crystal Data } \\
\hline Chemical Formula & $\mathrm{C}_{28} \mathrm{H}_{20} \mathrm{O}_{6} \mathrm{C}_{2} \mathrm{H}_{3} \mathrm{~N}$ \\
\hline$M_{\mathrm{r}}$ & 493.49 \\
\hline Crystal system, space group & Triclinic, $P-1$ \\
\hline Temperature (K) & 296 \\
\hline $\mathrm{a}, \mathrm{b}, \mathrm{c}(\AA)$ & $7.4588(3), 9.3676(4), 18.8953(8)$ \\
\hline$\alpha, \beta, \gamma\left({ }^{\circ}\right)$ & $84.535(3), 80.467(3), 73.215(3)$ \\
\hline$V\left(\AA^{3}\right)$ & $1244.96(9)$ \\
\hline Z & 2 \\
\hline Radiation type & Mo $\mathrm{K} \alpha$ \\
\hline$\mu\left(\mathrm{mm}^{-1}\right)$ & 0.09 \\
\hline Crystal size (mm) & $0.2 \times 0.18 \times 0.18$ \\
\hline \multicolumn{2}{|l|}{ Data collection } \\
\hline Diffractometer & Bruker Smart APEX CCD detector diffractometer \\
\hline Absorption correction & Multi-scan \\
\hline$T_{\min }, T_{\max }$ & $0.982,0.984$ \\
\hline $\begin{array}{l}\text { No. of measured, independent and observed } \\
{[I>2 \sigma(I)] \text { reflections }}\end{array}$ & $19318,4628,3118$ \\
\hline$R_{\text {int }}$ & 0.034 \\
\hline$(\sin \theta / \lambda)_{\max }\left(\AA^{-1}\right)$ & 0.606 \\
\hline \multicolumn{2}{|l|}{ Refinement } \\
\hline $\mathrm{R}\left[\mathrm{F}^{2}>2 \sigma\left(\mathrm{F}^{2}\right)\right], w \mathrm{R}\left(\mathrm{F}^{2}\right), \mathrm{S}$ & $0.051,0.140,1.05$ \\
\hline No. of reflections & 4628 \\
\hline No. of parameters & 337 \\
\hline No. of restraints & 0 \\
\hline H-atom treatment & H-atom parameters constrained \\
\hline$\Delta_{\max }, \Delta_{\min }\left(\mathrm{e} \AA^{-3}\right)$ & $0.21,-0.17$ \\
\hline
\end{tabular}


Robustness of the present methodology has been extended to other aromatic aldehydes and 4bromomethyl coumarins to obtain various compounds 5a-5m (Table 2). Then we turned our attention to use 4-hydroxy carbostyril 4 in this reaction. Direct application of the optimized reaction conditions resulted in the formation of isosteric compounds $5 \mathrm{n}-5 \mathrm{t}$ (Table 2 ).

Table 2. Physical data for compounds 5a-t.

\begin{tabular}{|c|c|c|c|c|c|}
\hline Compd. & $\mathbf{R}^{\prime}$ & $\mathbf{R}$ & Yield $(\%)^{\mathrm{a}}$ & m.p ${ }^{\mathrm{b}}\left({ }^{\circ} \mathrm{C}\right)$ & $\operatorname{Mass}(\mathbf{m} / \mathbf{z})^{\mathrm{c}}$ \\
\hline $5 a$ & $-\mathrm{H}$ & $6-\mathrm{OCH}_{3}$ & 84 & $240-242$ & 438 \\
\hline $5 \mathrm{~b}$ & $4-\mathrm{Cl}$ & 7- $\mathrm{CH}_{3}$ & 87 & $260-262$ & $456 / 458$ \\
\hline $5 c$ & $4-\mathrm{OCH}_{3}$ & $7,8-\mathrm{CH}_{3}$ & 83 & $248-250$ & 466 \\
\hline $5 \mathrm{~d}$ & $-\mathrm{H}$ & $7,8-\mathrm{CH}_{3}$ & 88 & $272-274$ & 436 \\
\hline $5 e$ & $4-\mathrm{Cl}$ & $7,8-\mathrm{CH}_{3}$ & 84 & $242-244$ & $470 / 472$ \\
\hline $5 f$ & $2-\mathrm{Cl}$ & $6-\mathrm{CH}_{3}$ & 82 & $237-239$ & $456 / 458$ \\
\hline $5 \mathrm{~g}$ & $4-\mathrm{NO}_{2}$ & $6-\mathrm{CH}_{3}$ & 87 & $265-267$ & 467 \\
\hline $5 \mathrm{~h}$ & $2-\mathrm{Cl}$ & 7- $\mathrm{CH}_{3}$ & 82 & $238-240$ & $456 / 458$ \\
\hline $5 \mathrm{i}$ & $-\mathrm{H}$ & 7- $\mathrm{CH}_{3}$ & 89 & 192-194 & 422 \\
\hline $5 \mathrm{j}$ & $-\mathrm{H}$ & $6-\mathrm{CH}_{3}$ & 85 & $278-280$ & 422 \\
\hline $5 \mathrm{k}$ & $4-\mathrm{OCH}_{3}$ & $6-\mathrm{CH}_{3}$ & 86 & $230-232$ & 452 \\
\hline 51 & $4-\mathrm{OCH}_{3}$ & 7- $\mathrm{CH}_{3}$ & 88 & $231-233$ & 452 \\
\hline $5 \mathrm{~m}$ & 4- $\mathrm{Cl}$ & $6-\mathrm{CH}_{3}$ & 84 & $276-278$ & $456 / 458$ \\
\hline $5 n$ & $2-\mathrm{Cl}$ & $6-\mathrm{CH}_{3}$ & 87 & $>300$ & $456 / 458$ \\
\hline 50 & $4-\mathrm{OCH}_{3}$ & 7- $\mathrm{CH}_{3}$ & 86 & $>300$ & 452 \\
\hline $5 p$ & $-\mathrm{H}$ & $7-\mathrm{CH}_{3}$ & 89 & $>300$ & $421(\mathrm{M}-\mathrm{H})$ \\
\hline $5 q$ & $4-\mathrm{OCH}_{3}$ & $6-\mathrm{CH}_{3}$ & 85 & $290-292$ & 451 \\
\hline $5 r$ & $4-\mathrm{OCH}_{3}$ & $6-\mathrm{Cl}$ & 87 & $261-263$ & $471 / 473$ \\
\hline $5 \mathrm{~s}$ & $4-\mathrm{OCH}_{3}$ & $6-\mathrm{OCH}_{3}$ & 89 & $270-272$ & 467 \\
\hline $5 \mathrm{t}$ & $4-\mathrm{OCH}_{3}$ & $7,8-\mathrm{CH}_{3}$ & 86 & $290-292$ & 465 \\
\hline
\end{tabular}

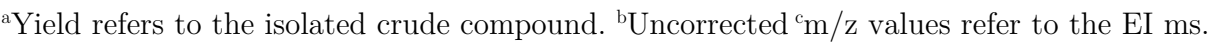

Based on our observations a plausible mechanistic pathway (Figure 3) is proposed. 4-bromomethyl coumarins 1 would form a carbanion stabilized as a pyridinium ylide A and an enolate B, which explains the ease with which this reactive species can be generated. Aromatic aldehydes 2 would react with 4hydroxy coumarin/quinoline 3/4 to form the Knoevenagel adduct C. Nucleophilic addition of B at the $\beta$-position of $\mathrm{C}$, would generate an enolate $\mathrm{D}$ in a typical Michael reaction. $\mathrm{A}$ back side nucleophilic attack on to the carbon bearing pyridine as the leaving group leads to the formation of compounds 5 with dihydrofuran moiety which probably explains the observed trans stereochemistry. All the three steps in this pathway occur in a tandem reaction. It is interesting to note that the electrophilic carbon in compounds lacts as a nucleophile in the Micheal addition step and the overall three component reaction sequence a $1+3+1$ approach for the construction of five membered rings.

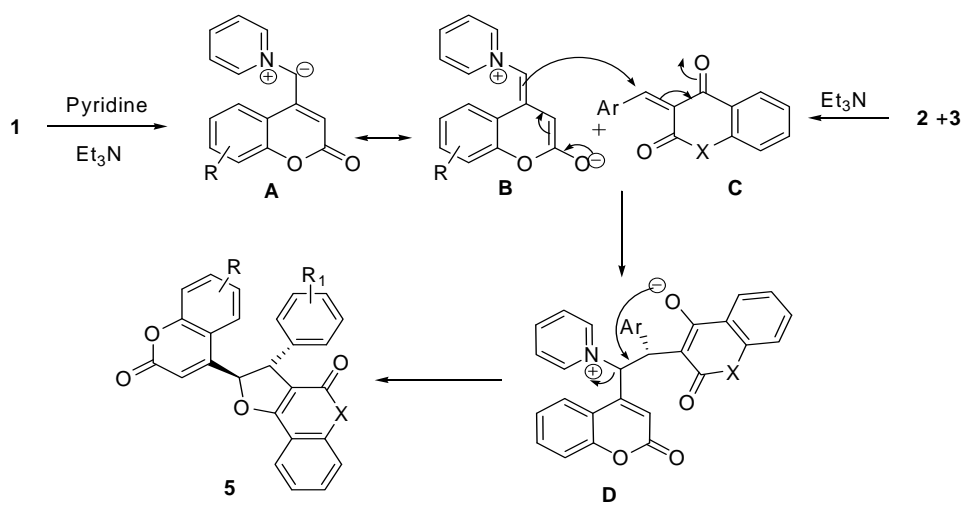

Figure 3. Plausible mechanistic pathway for the formation of furo quinolones and furo coumarins 4. 


\section{$3 \quad$ Experimental}

\subsection{General Methods}

Melting points were determined in open capillaries and are uncorrected. Infrared spectra were recorded on a Nicolet (FT-IR) spectrometer ( $\mathrm{v}$ max in cm-1), 1H (400 MHz), 13C (100 MHz) were recorded on a Bruker $(400 \mathrm{MHz})$ spectrometer.

3.2 General Procedure for Synthesis of trans-2,3-dihydro-2-(2-oxo-2H-chromen-4-yl)-3phenylfuro[3,2-c] chromen-4-ones/ quinolin-4(5H)-ones (5a-t)

A mixture of benzaldehyde (0.01mol, 1g), 4-hydroxy-2H-chromen-2-one/quinolin-4(5H)-ones (0.01mol, $1.26 \mathrm{~g})$, 4-(bromomethyl)-2H-chromen-2-one $(0.01 \mathrm{~mol})$, and pyridine $(0.03 \mathrm{~mol}, 2.37 \mathrm{~g})$ in acetonitrile (15 $\mathrm{mL}$ ) was taken in $100 \mathrm{ml}$ R.B flask fitted with condenser containing guard tube and reaction mixture was refluxed for $2 \mathrm{~h}$. Then triethylamine $(0.03 \mathrm{~mol}, 3.03 \mathrm{~g})$ was added and resulting reaction mixture was refluxed for $12 \mathrm{~h}$. Progress of the reaction was monitored by checking the TLC. After completion of the reaction, reaction mixture was cooled and obtained solid was filtered and dried. All the title compounds were further purified by crystallization in acetonitrile so as to get the pure product.

\subsection{Physical and Spectral Data}

\section{3a: 2,3-dihydro-2-(6-methoxy-2-oxo-2H-chromen-4-yl)-3-phenylfuro[3,2-c]chromen-4-one} (5a).

Colorless solid (acetonitrile), mp 240-242 oC, yield 84\%; IR (KBr, v in cm -1): $1722(-\mathrm{C}=\mathrm{O}) .1 \mathrm{H}-$ NMR (400 MHz, DMSO): 3.38 (s, 3H, -OCH3), 4.60 (d, 1H, J = 5.6 Hz, -CH-), 6.43 (s, 1H, C3-H), 6.51 $(\mathrm{d}, 1 \mathrm{H}, \mathrm{J}=2.8 \mathrm{~Hz}, \mathrm{Ar}-\mathrm{H}), 6.69(\mathrm{~d}, 1 \mathrm{H}, \mathrm{J}=5.6 \mathrm{~Hz},-\mathrm{CH}-), 7.21$ (dd, $1 \mathrm{H}, \mathrm{J}=2.8,9.2 \mathrm{~Hz}, \mathrm{Ar}-\mathrm{H}), 7.35-$ 7.37 (m, 1H, Ar-H), 7.40-7.42 (m, 5H, Ar-H), 7.47-7.52 (m, 2H, Ar-H), 7.74-7.79 (m, 1H, Ar-H), 7.98 (dd, $1 \mathrm{H}, \mathrm{J}=1.2,7.6 \mathrm{~Hz}, \mathrm{Ar}-\mathrm{H}), \mathrm{m} / \mathrm{z}=438.13 \mathrm{C}-\mathrm{NMR}: 52.6,54.9,89.1,105.3,107.1,110.9,111.5,116.2$, 116.6, 118.0, 120.2, 123.1, 124.5, 127.8, 128.1, 128.8, 133.3, 139.5, 147.8, 151.9, 154.7, 155.1, 158.1, 159.6, 164.9. $\mathrm{MS} \mathrm{m} / \mathrm{z}=438$. Anal. calc. for C27H18O6: C, 73.97; H, 4.14. Found: C, 73.92; H, 4.16.

3.3b: 3-(4-chlorophenyl)-2,3-dihydro-2-(7-methyl-2-oxo-2H-chromen-4-yl)furo[3,2c]chromen-4-one (5b).

Colorless solid (acetonitrile), mp 260-262 oC, yield 87\%; IR (KBr, v in cm -1): $1724(-\mathrm{C}=\mathrm{O}) .1 \mathrm{H}-$ NMR (400 MHz, DMSO): 2.40 (s, 3H, CH3), 4.63 (d, 1H, J = 5.6 Hz, -CH-), 6.37 (s, 1H, C3-H), 6.59 $(\mathrm{dd}, 1 \mathrm{H}, \mathrm{J}=0.8,5.6 \mathrm{~Hz},-\mathrm{CH}-), 7.03$ (d, 1H, J = 8.4 Hz, Ar-H), 7.10 (d, 1H, J = 8.4 Hz, Ar-H), 7.31 (s, 1H, Ar-H), 7.37-7.39 (m, 2H, Ar-H), 7.44-7.7.53 (m, 4H, Ar-H), 7.75-7.79 (m, 1H, Ar-H), 7.94 (dd, 1H, J $=1.6,8.0 \mathrm{~Hz}$ ). 13C-NMR: 20.9, 51.7, 89.2, 105.0, 110.1. 11.6, 113.5, 116.7, 117.1, 123.1, 124.5, 124.6, $125.3,128.8,129.9,132.4,133.4,138.5,143.4,151.7,153.7,154.8,158.2,159.7,165.3 . \mathrm{MS} \mathrm{m} / \mathrm{z}=456(\mathrm{M})$, 458(M+2). Anal. calc. for C27H17ClO5: C, 70.98; H, 3.75. Found: C, 70.97; H, 3.73.

3.3c: 2,3-dihydro-3-(4-methoxyphenyl)-2-(7,8-dimethyl-2-oxo-2H-chromen-4-yl)furo[3,2c]chromen-4-one (5c).

Colorless solid (acetonitrile), mp 248-250 oC, yield 83\%; IR (KBr, v in cm -1): $1719(-\mathrm{C}=\mathrm{O})$. $1 \mathrm{H}-$ NMR (400 MHz, DMSO): 2.29 (s, 3H, CH3), 2.23 (s, 3H, CH3), 3.76 (s, 3H, -OCH3), 4.48 (d, $1 \mathrm{H}, \mathrm{J}=$ $5.6 \mathrm{~Hz},-\mathrm{CH}-), 6.32$ (d, 1H, J = 0.8 Hz, C3-H), 6.52 (d, 1H, J = 5.6 Hz), 6.88 (d, 1H, J = 8.4, -CH-), 6.93-6..96 (m, 2H, Ar-H), 7.07 (d, 1H, J = 8Hz), 7.23 (m, 2H, Ar-H), 7.46-7.52 (m, 2H, Ar-H), 7.74-7.78 (m, 1H, Ar-H), 7.95 (dd, 1H, J = 1.6, 8 Hz). 13C-NMR: 11.2, 19.8, 52.0, 55.1, 105.5, 109.2, 111.6, 113.6, 114.2, 116.7, 121.6, 123.1, 124.3, 124.5, 125.3, 129.0, 131.5, 133.3, 141.9, 151.6, 152.5, 154.7, 158.2, 158.8, 159.7, 164.8. MS m/z= 466. Anal. calc. for C29H22O6: C, 74.67; H, 4.75. Found: C, 74.64; H, 4.76.

3.3d: 2,3-dihydro-2-(7,8-dimethyl-2-oxo-2H-chromen-4-yl)-3-phenylfuro[3,2-c]chromen-4one $(5 \mathrm{~d})$.

Colorless solid (acetonitrile), mp 272-274 oC, yield 88\%; IR (KBr, v in cm -1): $1705(-\mathrm{C}=\mathrm{O}) .1 \mathrm{H}-$ NMR (400 MHz, DMSO): 2.229 (s, 3H, CH3), 2.35 (s, 3H, CH3), 4.53 (d, 1H, J = 5.6 Hz, -CH-), 6.35 (d, $1 \mathrm{H}, \mathrm{J}=0.8 \mathrm{~Hz}, \mathrm{C} 3-\mathrm{H}), 6.59(\mathrm{dd}, 1 \mathrm{H}, \mathrm{J}=0.8,5.6 \mathrm{~Hz},-\mathrm{CH}-), 6.85$ (d, 1H, J = 8.4 Hz, Ar-H), 7.05 (d, $1 \mathrm{H}$, 
$\mathrm{J}=8.0 \mathrm{~Hz}, \mathrm{Ar}-\mathrm{H}), 7.33-7.53(\mathrm{~m}, 7 \mathrm{H}, \mathrm{Ar}-\mathrm{H}), 7.74-7.79(\mathrm{~m}, 1 \mathrm{H}, \mathrm{Ar}-\mathrm{H}), 7.96(\mathrm{dd}, 1 \mathrm{H}, \mathrm{J}=1.2,7.6 \mathrm{~Hz}) . \mathrm{MS}$ $\mathrm{m} / \mathrm{z}=436$. Anal. calc. for C28H20O5: C, 77.05; H, 4.62. Found: C, 77.03; H, 4.64.

3.3e: 3-(4-chlorophenyl)-2,3-dihydro-2-(7,8-dimethyl-2-oxo-2H-chromen-4-yl)furo[3,2c]chromen-4-one (5e).

Colorless solid (acetonitrile), mp 242-244 oC, yield 84\%; IR (KBr, v in cm -1): $1716(-\mathrm{C}=\mathrm{O})$. $1 \mathrm{H}-$ NMR (400 MHz, DMSO): 2.30 (s, 3H, CH3), 2.35 (s, 3H, CH3), 4.60 (d, 1H, J = 5.6 Hz, -CH-), 6.36 (d, $1 \mathrm{H}, \mathrm{J}=0.8 \mathrm{~Hz}, \mathrm{C} 3-\mathrm{H}), 6.59$ (dd, 1H, J = 1.2, $6.0 \mathrm{~Hz},-\mathrm{CH}-), 6.87$ (d, 1H, J = 8.4 Hz, Ar-H), 7.08 (d, $1 \mathrm{H}$, $\mathrm{J}=8.4 \mathrm{~Hz}, \mathrm{Ar}-\mathrm{H}), 7.35-7.53(\mathrm{~m}, 6 \mathrm{H}, \mathrm{Ar}-\mathrm{H}), 7.75-7.79(\mathrm{~m}, 1 \mathrm{H}, \mathrm{Ar}-\mathrm{H}), 7.95$ (dd, 1H, J = 1.6, 8.0 Hz). MS $\mathrm{m} / \mathrm{z}=470$. Anal. calc. for $\mathrm{C} 28 \mathrm{H} 19 \mathrm{ClO} 5: \mathrm{C}, 71.42 ; \mathrm{H}, 4.07$. Found: C, 71.44; H, 4.05.

3.3f: 3-(2-chlorophenyl)-2,3-dihydro-2-(7,8-dimethyl-2-oxo-2H-chromen-4-yl)furo[3,2c]chromen-4-one (5f).

Colorless solid (acetonitrile), mp 236-238 oC, yield 82\%; IR (KBr, v in cm -1): $1729(-\mathrm{C}=\mathrm{O})$. 1HNMR (400 MHz, DMSO): 2.07 (s, 3H, CH3), 2.297 (s, 3H, CH3), 4.76 (d, 1H, J = 5.6 Hz, -CH-), 6.37 (d, $1 \mathrm{H}, \mathrm{J}=1.2 \mathrm{~Hz}, \mathrm{C} 3-\mathrm{H}), 6.47(\mathrm{~d}, 1 \mathrm{H}, \mathrm{J}=2.8 \mathrm{~Hz}, \mathrm{Ar}-\mathrm{H}), 6.52(\mathrm{~m}, 1 \mathrm{H}, \mathrm{Ar}-\mathrm{H}), 6.68(\mathrm{dd}, 1 \mathrm{H}, \mathrm{J}=1.2,5.6$ $\mathrm{Hz},-\mathrm{CH}-), 7.15$ (s, 1H, Ar-H), 7.37 (d, 1H, J = 8.4, Ar-H), 7.47-7.55 (m, 3H, Ar-H), 7.74-7.80 (m, 2H, Ar-H), $7.96(\mathrm{dd}, 1 \mathrm{H}, \mathrm{J}=1.6,8.0 \mathrm{~Hz}) . \mathrm{MS} \mathrm{m} / \mathrm{z}=470$. Anal. calc. for C28H19ClO5: C, 71.42; H, 4.07. Found: C, 71.38; H, 3.72.

3.3g: 2,3-dihydro-2-(6-methyl-2-oxo-2H-chromen-4-yl)-3-(4-nitrophenyl)furo[3,2-c]chromen4-one $(5 \mathrm{~g})$.

Colorless solid (acetonitrile), mp 265-267 oC, yield 87\%; IR (KBr, v in cm -1): $1727(-\mathrm{C}=\mathrm{O}) .1 \mathrm{H}-$ NMR (400 MHz, DMSO): 2.130 (s, 3H, CH3), 5.29 (d, 1H, J = 4.0 Hz, -CH-), 6.57 (s, 1H, C3-H), 6.77 $(\mathrm{d}, 1 \mathrm{H}, \mathrm{J}=4.0 \mathrm{~Hz},-\mathrm{CH}-), 6.98(\mathrm{~s}, 1 \mathrm{H}, \mathrm{Ar}-\mathrm{H}), 7.37(\mathrm{~d}, 1 \mathrm{H}, \mathrm{J}=8.4 \mathrm{~Hz}, \mathrm{Ar}-\mathrm{H}), 7.45-7.79(\mathrm{~m}, 7 \mathrm{H}, \mathrm{Ar}-\mathrm{H})$, $7.91(\mathrm{~d}, 1 \mathrm{H}, \mathrm{J}=7.2 \mathrm{~Hz}, \mathrm{Ar}-\mathrm{H}), 8.03(\mathrm{~d}, 1 \mathrm{H}, \mathrm{J}=8.4 \mathrm{~Hz})$. MS m/z = 451(M-16). Anal. calc. for C27H17NO7: C, 69.38; H, 3.67; N, 3.00. Found: C, 69.36; H, 3.68; N, 3.02.

3.3h: 3-(2-chlorophenyl)-2,3-dihydro-2-(7-methyl-2-oxo-2H-chromen-4-yl)furo[3,2c]chromen-4-one $(5 \mathrm{~h})$.

Colorless solid (acetonitrile), mp 238-240 oC, yield 82\%; IR (KBr, v in cm -1): 1735(-C=O). $1 \mathrm{H}-$ NMR (400 MHz, DMSO): 2.45 (s, 3H, CH3), 5.07 (d, 1H, J = 5.4 Hz, -CH-), 6.21 (d, 1H, J = 5.4 Hz, CH-), 6.44 (s, 1H, C3-H), 6.91-7.70 (m, 10H, Ar-H), 7.86 (d, 1H, J = 7.2 Hz). m/z: 456. Anal.calc. for C27H17ClO5: C, 70.98; H, 3.75. Found: C, 70.96; H, 3.77.

3.3i: 2,3-dihydro-2-(7-methyl-2-oxo-2H-chromen-4-yl)-3-phenylfuro[3,2-c]chromen-4-one (5i).

Colorless solid (acetonitrile), mp 192-194 oC, yield 89\%; IR (KBr, v in cm -1): $1719(-\mathrm{C}=\mathrm{O}) .1 \mathrm{H}-$ NMR (400 MHz, DMSO): 2.32 (s, 3H, CH3), 4.32 (d, 1H, J = 4.0 Hz, -CH-), 6.11 (d, 1H, J = 4.0 Hz, CH-), 6.21 (s, 1H, C3-H), 6.83-6.88 (m, 2H, Ar-H), 7.07 (s, 1H, Ar-H), 7.20-7.31 (m, 7H, Ar-H), 7.53$7.56(\mathrm{~m}, 1 \mathrm{H}, \mathrm{Ar}-\mathrm{H}), 7.76(\mathrm{~d}, 1 \mathrm{H}, \mathrm{J}=4.5 \mathrm{~Hz}) . \mathrm{MS} \mathrm{m} / \mathrm{z} 422$. Anal. calc. for C27H18O5: C, 76.77; H, 4.29. Found: C, 76.74; H, 4.25 .

3.3j: 2,3-dihydro-2-(6-methyl-2-oxo-2H-chromen-4-yl)-3-phenylfuro[3,2-c]chromen-4-one (5j) Colorless solid (acetonitrile), mp 278-280 oC, yield 85\%; IR (KBr, v in $\mathrm{cm} \mathrm{-1):} 1734(-\mathrm{C}=\mathrm{O})$. $1 \mathrm{H}-$ NMR (400 MHz, DMSO): 2.20 (s, 3H, CH3), 4.47 (d, 1H, J = 4.8 Hz, -CH-), 6.22 (d, 1H, J = 4.5 Hz, CH-), 6.46 (s, 1H, C3-H), $6.82(\mathrm{~s}, 1 \mathrm{H}, \mathrm{Ar}-\mathrm{H}), 7.26-7.44$ (m, 9H, Ar-H), 7.67 (t, 1H, J = 7.5 Hz, Ar-H), $7.90(\mathrm{~d}, 1 \mathrm{H}, \mathrm{J}=7.5 \mathrm{~Hz}$ ). MS m/z 422. Anal. calc. for C27H18O5: C, 76.77; H, 4.29. Found: C, 76.76; H, 4.30 .

3.3k: 2,3-dihydro-3-(4-methoxyphenyl)-2-(6-methyl-2-oxo-2H-chromen-4-yl)furo[3,2c]chromen-4-one (5k).

Colorless solid (acetonitrile), mp 230-232 oC, yield 86\%; IR (KBr, v in cm -1): $1722(-\mathrm{C}=\mathrm{O})$. $1 \mathrm{H}-$ NMR (400 MHz, DMSO): 2.24 (s, 3H, CH3), 3.83 (s, 3H, -OCH3), 4.44 (d, 1H, J = 4.8 Hz, -CH-),6.17 $(\mathrm{d}, 1 \mathrm{H}, \mathrm{J}=4.5 \mathrm{~Hz},-\mathrm{CH}-), 6.44(\mathrm{~s}, 1 \mathrm{H}, \mathrm{C} 3-\mathrm{H}), 6.87$ (s, 1H, Ar-H), 6.96 (d, 2H, J = 8.4 Hz, Ar-H), 7.26$7.44(\mathrm{~m}, 6 \mathrm{H}, \mathrm{Ar}-\mathrm{H}), 7.64-7.69$ (m, 1H, Ar-H), 7.89 (d, 1H, J = 7.8 Hz, Ar-H). MS m/z 451 (M-1). Anal. calc. for C28H20O6: C, 74.33; H, 4.46. Found: C, 74.32; H, 4.48.

3.31: 2,3-dihydro-3-(4-methoxyphenyl)-2-(7-methyl-2-oxo-2H-chromen-4-yl)furo[3,2c]chromen-4-one (5l).

Colorless solid (acetonitrile), mp 231-233 oC, yield 88\%; IR (KBr, v in cm -1): $1716(-\mathrm{C}=\mathrm{O})$. $1 \mathrm{H}-$ NMR (400 MHz, DMSO): 2.45 (s, 3H, CH3), 3.83 (s, 3H, -OCH3), 4.43 (d, 1H, J = 4.5 Hz, -CH-), 6.17 $(\mathrm{d}, 1 \mathrm{H}, \mathrm{J}=4.5 \mathrm{~Hz},-\mathrm{CH}-), 6.36(\mathrm{~s}, 1 \mathrm{H}, \mathrm{C} 3-\mathrm{H}), 6.94$ (d, 2H, J = 8.7 Hz, Ar-H), 7.01 (s, 2H, Ar-H), 7.22- 
$7.44(\mathrm{~m}, 5 \mathrm{H}, \mathrm{Ar}-\mathrm{H})$, 7.63-7.69 (m, 1H, Ar-H), 7.87 (d, 1H, J = 7.5 Hz). MS m/z 452. Anal. calc. for C28H20O6: C, 74.33; H, 4.46. Found: C, 74.37; H, 4.44.

3.3m: 3-(4-chlorophenyl)-2,3-dihydro-2-(6-methyl-2-oxo-2H-chromen-4-yl)furo[3,2c]chromen-4-one $(5 \mathrm{~m})$.

Colorless solid (acetonitrile), mp 276-278 oC, yield 84\%; IR (KBr, v in $\mathrm{cm} \mathrm{-1):} 1715(-\mathrm{C}=\mathrm{O}) .1 \mathrm{H}-$ NMR (400 MHz, DMSO): 2.24 (s, 3H, CH3), 4.46 (d, 1H, J = 4.8 Hz, -CH-), 6.17 (dd, 1H, J = 0.9, 4.8 $\mathrm{Hz},-\mathrm{CH}-), 6.44$ (s, 1H, C3-H), 6.78 (s, 1H, Ar-H), 7.26-7.45 (m, 8H, Ar-H), 7.65-7.71 (m, 1H, Ar-H), 7.89 (d, $1 \mathrm{H}, \mathrm{J}=8.1 \mathrm{~Hz}$ ). MS m/z 456. Anal. calc. for C27H17ClO5: C, 70.98; H, 3.75. Found: C, 70.92; $\mathrm{H}, 3.76$.

3.3n: 3-(2-chlorophenyl)-2,3-dihydro-2-(6-methyl-2-oxo-2H-chromen-4-yl)furo[3,2c] quinolin-4(5H)-one $(5 \mathrm{n})$.

Colorless solid (acetonitrile), mp $>300 \mathrm{oC}$, yield 87\%; IR (KBr, v in $\mathrm{cm} \mathrm{-1):} 1704(-\mathrm{C}=\mathrm{O}), 3368(-$ OH). 1H-NMR (400 MHz, DMSO): 2.15 (s, 3H, CH3), 4.94 (d, 1H, J = 5.6 Hz, -CH-), 6.37 (d, 1H, J = $1.2 \mathrm{~Hz}, \mathrm{C} 3-\mathrm{H}), 6.49$ (dd, 1H, J = 1.2, $5.6 \mathrm{~Hz}, \mathrm{Ar}-\mathrm{H}), 6.84$ (s, 1H, Ar-H), 7.28-7.64 (m, 9H, Ar-H), 7.89 (d, $1 \mathrm{H}, \mathrm{J}=6.8 \mathrm{~Hz}), 11.54(\mathrm{~s}, 1 \mathrm{H},-\mathrm{NH}) . \mathrm{MS} \mathrm{m} / \mathrm{z}=455$. Anal. calc. for C27H18ClNO4: C, 71.13; H, 3.98; N, 3.07. Found: C, 71.13; H, 3.97; N, 3.04 .

3.3o: 2,3-dihydro-3-(4-methoxyphenyl)-2-(7-methyl-2-oxo-2H-chromen-4-yl)furo[3,2c] quinolin-4(5H)-one (5o).

Colorless solid (acetonitrile), mp $>300$ oC, yield 86\%; IR (KBr, v in cm -1): $1736(-\mathrm{C}=\mathrm{O}), 3431(-$ OH). 1H-NMR (400 MHz, DMSO): 2.41 (s, 3H, CH3), 3.76 (s, 3H, -OCH3), 4.40 (d, 1H, J = 4.0 Hz, CH-), $6.18(\mathrm{~d}, 1 \mathrm{H}, \mathrm{J}=0.8 \mathrm{~Hz}, \mathrm{C} 3-\mathrm{H}), 6.38(\mathrm{~d}, 1 \mathrm{H}, \mathrm{J}=4.0 \mathrm{~Hz}), 6.94(\mathrm{~d}, 2 \mathrm{H}, \mathrm{J}=8.8 \mathrm{~Hz}, \mathrm{Ar}-\mathrm{H}), 7.11(\mathrm{~s}$, $2 \mathrm{H}, \mathrm{Ar}-\mathrm{H}), 7.20(\mathrm{~d}, 2 \mathrm{H}, \mathrm{J}=8.4 \mathrm{~Hz}), 7.27-7.40(\mathrm{~m}, 3 \mathrm{H}, \mathrm{Ar}-\mathrm{H}), 7.58-7.62(\mathrm{~m}, 1 \mathrm{H}, \mathrm{Ar}-\mathrm{H}), 7.86(\mathrm{~d}, 1 \mathrm{H}, \mathrm{J}=$ $7.2 \mathrm{~Hz}), 11.51(\mathrm{~s}, 1 \mathrm{H},-\mathrm{NH})$. MS m/z = 455. Anal. calc. for C28H21NO5: C, 74.49; H, 4.69; N, 3.10. Found: C, 74.48; H, 4.67; N, 3.12.

3.3p: 2,3-dihydro-2-(7-methyl-2-oxo-2H-chromen-4-yl)-3-phenylfuro[3,2-c]quinolin-4(5H)one $(5 \mathrm{p})$.

Colorless solid (acetonitrile), mp >300 oC, yield 89\%; IR (KBr, v in cm -1): $1736(-\mathrm{C}=\mathrm{O}), 3399(-$ OH). 1H-NMR (400 MHz, DMSO): 2.41 (s, 3H, CH3), 4.46 (d, 1H, J = 4.0 Hz, -CH-), 6.20 (s, 1H, C3$\mathrm{H}), 6.45$ (d, 1H, J = 4.0 Hz, -CH-), 7.09 (s, 1H, Ar-H), 7.29-7.41(m, 9H, Ar-H), 7.61 (t, 1H, J = 8.0 Hz, Ar-H), 7.88 (d, 1H, J = 8.0 Hz), 11.53 (s, 1H, NH). MS m/z 421. Anal. calc. for C27H19NO4: C, 76.95; H, 4.54; N, 3.32. Found: C, 76.96; H, 4.50; N, 3.33.

3.3q: 2,3-dihydro-3-(4-methoxyphenyl)-2-(6-methyl-2-oxo-2H-chromen-4-yl)furo[3,2c] quinolin-4(5H)-one $(5 \mathrm{q})$.

Colorless solid (acetonitrile), mp 290-292 oC, yield 85\%; IR (KBr, v in cm -1): 1730 (-C=O), $3363(-$ OH). 1H-NMR (400 MHz, DMSO): 2.20 (s, 3H, CH3), 3.77 (s, 3H, -OCH3), 4.41 (d, 1H, J = 4.0 Hz, CH-), 6.26 (s, 1H, C3-H), 6.37 (d, 1H, J = 4.0 Hz, -CH-), 6.95-6.98 (m, 3H, Ar-H), 7.20-7.63 (m, 7H, Ar$\mathrm{H}), 7.89(\mathrm{~d}, 1 \mathrm{H}, \mathrm{J}=8.0 \mathrm{~Hz}), 11.51(\mathrm{~s}, 1 \mathrm{H}, \mathrm{NH})$. MS m/z 451. Anal. calc. for C28H21NO5: C, 74.49; H, $4.69 ;$ N, 3.10. Found: C, 74.47; H, 4.68; N, 3.11.

3.3r: 2-(6-chloro-2-oxo-2H-chromen-4-yl)-2,3-dihydro-3-(4-methoxyphenyl)furo[3,2c]quinolin-4(5H)-one (5r).

Colorless solid (acetonitrile), mp 260-262 oC, yield 87\%; IR (KBr, v in cm -1): 1737 (-C=O), $3472(-$ $\mathrm{OH})$. 1H-NMR (400 MHz, DMSO): 3.76 (s, 3H, -OCH3), 4.47 (d, 1H, J = 4.0 Hz, -CH-), $6.34(\mathrm{~s}, 1 \mathrm{H}$, C3-H), 6.43 (d, 1H, J = 4.0 Hz, -CH-), 6.96 (d, 2H, J = 8.0 Hz, Ar-H), 7.17-7.72 (m, 8H, Ar-H), 7.88 (d, $1 \mathrm{H}, \mathrm{J}=8.0 \mathrm{~Hz}), 11.52(\mathrm{~s}, 1 \mathrm{H}, \mathrm{NH})$. MS m/z 471. Anal. calc. forC27H18ClNO5:C, 68.72; H, 3.84; Cl, 7.51; N, 2.97. Found: C, 68.70; H, 3.86; Cl, 7.53; N, 2.96 .

3.3s: 2,3-dihydro-2-(6-methoxy-2-oxo-2H-chromen-4-yl)-3-(4-methoxyphenyl)furo[3,2c]quinolin-4 $(5 \mathrm{H})$-one $(5 \mathrm{~s})$

Colorless solid (acetonitrile), mp 270--272 oC, yield 89\%; IR (KBr, v in cm -1): 1729 (-C=O), $3363(-$ OH). 1H-NMR (400 MHz, DMSO): 3.49 (s, 3H, -OCH3), 3.75 (s, 3H, -OCH3), 4.45 (d, 1H, J = 4.0 Hz, CH-), 6.27 (s, 1H, C3-H), 6.46 (d, 1H, J = 4.0 Hz, -CH-), 6.63 (d, 1H, J = 4.0 Hz, Ar-H), 6.94 (d, 2H, J $=12 \mathrm{~Hz}), 7.22-7.63(\mathrm{~m}, 7 \mathrm{H}, \mathrm{Ar}-\mathrm{H}), 7.88(\mathrm{~d}, 1 \mathrm{H}, \mathrm{J}=8.0 \mathrm{~Hz}), 11.51(\mathrm{~s}, 1 \mathrm{H}, \mathrm{NH}) . \mathrm{MS} \mathrm{m} / \mathrm{z} 467$. Anal. calc. forC28H21NO6: C, 71.94; H, 4.53; N, 3.00. Found: C, 71.92; H, 4.55; N, 3.01.

3.3t: 2,3-dihydro-3-(4-methoxyphenyl)-2-(7,8-dimethyl-2-oxo-2H-chromen-4-yl)furo[3,2c] quinolin-4(5H)-one (5t). 
Colorless solid (acetonitrile), mp 290-292 oC, yield 86\%; IR (KBr, v S in cm -1): 1733 (-C=O), 3392 (-OH). 1H-NMR (400 MHz, DMSO): 2.29 (s, 3H, CH3), 2.36 (s, 3H, CH3), 3.76 (s, 3H, -OCH3), 4.37 (d, $1 \mathrm{H}, \mathrm{J}=4.0 \mathrm{~Hz},-\mathrm{CH}-), 6.18(\mathrm{~s}, 1 \mathrm{H}, \mathrm{C} 3-\mathrm{H}), 6.37(\mathrm{~d}, 1 \mathrm{H}, \mathrm{J}=4.0 \mathrm{~Hz},-\mathrm{CH}-), 6.95(\mathrm{t}, 3 \mathrm{H}, \mathrm{J}=8.0 \mathrm{~Hz})$, 7.09-7.62 (m, 6H, Ar-H), $7.87(\mathrm{~d}, 1 \mathrm{H}, \mathrm{J}=8.0 \mathrm{~Hz}), 11.52(\mathrm{~s}, 1 \mathrm{H}, \mathrm{NH})$. MS m/z 465. Anal. calc. for C29H23NO5: C, 74.83; H, 4.98; N, 3.01. Found: C, 74.82; H, 4.96; N, 3.00.

\section{Conclusion}

We have shown a tandem Knoevenagel and Michael addition reactions sequence leading to the generation of isosteric tricyclic furo coumarins and azacoumarins. The reported compounds bear a close resemblence with the naturally occuring bio-active compounds. The ability of coumarins to form solvates with acetonitrile has also been recorded for the first time during this investigation.

Acknowledgement: The authors thank National Science Council, Taiwan for the financial assistance. One of the authors (Reshma.Naik) thanks the DST-New Delhi for the INSPIRE fellowship. We thank University Scientific Instrumentation Center (USIC), Karnatak University Dharwad for IR, NMR, MS and analytical data.

\section{References}

1. B. Ganem, "Strategies for Innovation in Multicomponent Reaction Design," Acc. Chem. Res, vol. 42, pp. 463-472, 2009.

2. C. DeGraaff, E. Ruijter and R. V. A. Orru, "Recent developments in asymmetric multicomponent reactions," Chem. Soc. Rev,vol. 41, pp. 3969-4009,2012.

3. B. B. Tourie and D. G. Hall, "Natural Product Synthesis Using Multicomponent Reaction Strategies" Chem. Rev, vol. 109, pp. 4439-4486, 2009.

4. J. E Biggs-Houk, A.Younai and J. T. Shaw, "Recent advances in multicomponent reactions for diversity-oriented synthesis," Curr. Opinion in Chem. Biol, vol. 14, pp. 371-382, 2010.

5. (a) A. Syamala, "Recent Progress in Three-Component Reactions: An Update," Org. Prep. Proc. Int, vol. 41, pp. 1-68,2009. (b) V. Nair, R. S. Menon and V. Sreekumar, "Multicomponent reactions based on nucleophilic carbenes and their applications in organic synthesis," Pure Appl. Chem, vol. 77, pp. 1191-1198, 2005.

6. D. Audisio, S. Messaoudi, J. D. Brion and M. Alami, "A Simple Synthesis of Functionalized 3-Bromocoumarins by a One-Pot Three-Component Reaction,"Eur. J. Org. Chem, vol. 75, pp. 1046-1051, 2010.

7. T. Meng, Y. Zou, O. Khorev, Y. Jin, H. Zhou, Y. Zhang, D. Hu, L. Ma, X. Wang and J. Shena, "A Mild and Efficient Method for Large Scale Synthesis of 3-Aminocoumarins and Its Further Application for the Preparation of 4-Bromo-3-aminocoumarins," Adv. Synth. Catal, vol. 353, pp. 918-924, 2011.

8. M. Matache, C. Dobrota, N. C. Bogdan, I. Dumitru, L. L. Ruta, C. C. Paraschivescu, I. C. Farcsasnu, I. Baciu and D. P. Funeriu, "Synthesis of fused dihydro-pyrimido[4,3- $d]$ coumarins using Biginelli multicomponent reaction as key step," Tetrahedron,vol. 65, pp. 5949-5957,2009.

9. J .Wu, "General Microwave-assisted Protocols for the Expedient Synthesis of Furo[3,2-c]chromen-4-ones," Chem. Lett, vol. 35, pp. 118-119, 2006.

10. (a) A. Shabani, M. B. Teimouri, S. Samidi and K. Soleimani, "Microwave-Assisted Three-Component Condensation on Montmorillonite K10: Solvent Free Synthesis of Furopyrimidines, Furocoumarins, and Furopyranones," Synthetic. Commun, vol. 35, pp. 535-541,2005. (b) E. Altieri, M. Cordaro, G. Grassi, F. Ristano and A. Scala, 'Decorated 6,6', 7,7' -tetrahydro- $1 H, 1^{\prime} \quad H-2,3^{\prime}$-biindole scaffold as promising candidate for recognition of the CDK2 allosteric site," Tetrahedron, vol. 66, pp. 9493-9496, 2010.

11. A. T. Khan, M. Lal, S. Ali and M. M. Khan, "One-pot three-component reaction for the synthesis of pyran annulated heterocyclic compounds using DMAP as a catalyst," Tetrahedron Lett, vol. 52, pp. 5327-5332, 2011.

12. S. Balalaie, M. Bigdeli, E. Sheikhhosseinib, A. Habibib, H. P. Moghadamd and M .Naderid, "Efficient Synthesis of Novel Coumarin-3-carboxamides (2-Oxo-2H-1-benzopyran-3-carboxamides) Containing Lipophilic Spacers,"Helv. Chim.Acta, vol. 95, pp. 528-535, 2012. 
13. C. Che, S. Li, X. Jiang, J. Quan, S. Lin and Z. Yang, "One-Pot Syntheses of Chromeno[3,4-c]pyrrole-3,4-diones via Ugi-4CR and Intramolecular Michael Addition," Org. Let, vol. 12, pp. 4682-4685, 2010.

14. Md. N. Khan, S. Pal, S. Karamathulla and L. H. Choudhary, "Multicomponent reactions for facile access to coumarin-fused dihydroquinolines and quinolines: synthesis and photophysical studies,"New J. Chem, vol. 38, pp. 4722-4729, 2014.

15. V. Felipe, M. Thiago, R. Pereira and N. Castro, P. G. Guilherme, C. A. Cedric Stephan Graebinaband and A. E. $\mathrm{Ku}$ "mmerle, "Synthesis and mechanism of novel fluorescent coumarin-dihydropyrimidinone dyads obtained by the Biginelli multicomponent reaction,"New J. Chem, vol. 39, pp. 2323-2332, 2015.

16. M. Seydimemet, K. Ablajan, M. Hamdulla, W. Li, A. Omar, and M. Obul, "L-Proline catalyzed four-component one-pot synthesis of coumarin-containing dihydropyrano[2,3-c]pyrazoles under ultrasonic irradiation," Tetrahedron, vol. 72, pp. 7599-7605, 2016.

17. J. J. Jennings, C. P. Bhatt and A. K. Franz,"Lanthanum(III)-Catalyzed Three-Component Reaction of Coumarin-3-carboxylates for the Synthesis of Indolylmalonamides and Analysis of Their Photophysical Properties,"J. Org. Chem, vol. 81, pp. 6211-6222, 2016.

18. M. F. Grundon, "Quinoline, quinazoline, and acridone alkaloids," Nat. Prod. Rep, vol. 4, pp. 225-236, 1987.

19. (a) M. F. Grundon, "The Alkaloids," Nat. Prod. Rep, vol. 7, pp. 131-138, 1990. (b) J. P. Michael, "Quinoline, quinazoline and acridone alkaloids,"Nat.Prod. Rep,vol. vol. 14, pp. 605-618, 1997.

20. H. Irie, S. Uyeo and K. Yamamoto, "The structure of glaupalol, a novel furanocoumarin from Glaucidium palmatum Sieb. etZucc," Chem. Commun. pp. 547-548, 1967.

21. C. Giovanni, C. Giancarlo, P. Giovanni and A. Rita, "Synthesis of fercoprolone, a degraded prenylatedcoumarin," Tetrahedron, vol. 54, pp. 10819 -10826,1998.

22. K. Kojima, K. Isaka, P. Ondognii, O. Zevgeegiin, P. Gombosurengyin, K. Davgiin, H. Mizukami and Y. Ogihara, "Sesquiterpenoid Derivatives from Ferula ferulioides. IV," Chem. Pharm. Bul, vol. 48, pp. 353-356, 2000.

23. K. Duraipandiyan and C. Ignacimuthu, "Antibacterial and antifungal activity of Flindersine isolated from the traditional medicinal plant, Toddaliaasiatica (L.) Lam,"J. Ethnopharmacol, vol. 123, pp. 494-498, 2009.

24. L. K. Basco, S. Mitaku, A. L.Skaltsounis, N. N .Ravelomanantsoa, F. M. Tillequin, J .Koch, and Le. Bra, "In vitro activities of furoquinoline and acridone alkaloids against Plasmodium falciparum," Antimicrob.AgentsChemother, vol. 38, pp. 1169-1171, 1994.

25. H. Morita, T. Dota, and J. Kobayashi, "Antimitotic activity of glaupalol-related coumarins from Glaucidiumpalmatum," Bioorg.Med. Chem. Lett, vol. 14, pp. 3665-3668, 2004.

26. H. A. Oketch-Rabah, E. Lemmich, S. F. Dossaji, T. G. Theander, C. E. Olson, C. Cornett, A. Kharazmi, and S. B. Christensen, "Two New Antiprotozoal 5-Methylcoumarins from Vernoniabrachycalyx," J. Nat. Prod,vol. 60, pp. 458-461,1997.

27. (a) H. Suginome, K. Kobayashi, M. Itoh and S. Seko. "Handbook of Synthetic Photochemistry,"J. Org. Chem,vol. 55, pp. 4933-4943,1990. (b) H. Senboku, M. Takashima, M. Suzuki, K. Kobayashi and H. Suginome, "Photoinduced molecular transformations: Part 159. Formation of some furonaphthyridinones by selective $\beta$ scission of cyclobutanoxyl radicals generated from $[2+2]$ photoadducts of 4-hydroxy-1-phenyl $[1,8]$ naphthyridin2(1H)-one with alkenes," Tetrahedron, vol. 52, pp. 6125-6138, 1996.

28. H. Suginome, S. Seko, A. Konishi and K. Kobayashi, "One-step formation of furo[3,2-c]quinolin-4(5H)-ones by a new regioselective $[2+3]$ photoaddition of methoxy-substituted 4-hydroxyquinolin-2-one with alkenes," Tetrahedron. Lett,vol. 32, pp. 5115-5118, 1991.

29. Y. R. Lee, B. S. Kim and H. I.Kweon, "Efficient Synthesis of Dihydrofuroquinolinones and Furoquinolinones by Silver(I)/Celite Promoted Oxidative Cycloaddition," Tetrahedron, 56, 3867-3874, 2000.

30. D. R. Boyd, N. D. Sharma, S. A. Barr, J. G. Carroll, D. Makerracher and J. F. Malone, "Synthesis and absolute stereochemistry assignment of enantiopuredihydrofuro- and dihydropyrano-quinoline alkaloids," J. Chem. Soc., Perkin Trans,vol. 1, pp. 3397-3405, 2000.

31. F. Risitano, G. Grassi, F. Foti and C. Biardo, "A convenient synthesis of furo[3,2-c]coumarins by a tandem alkylation/intramolecular aldolisation reaction," Tetrahedron.Lett, vol. 42, pp. 3503-3505, 2001.

32. Y. R. Lee and J. Y. Suk, "Efficient synthesis of dihydrofurans and furans by rhodium(II)-catalyzed reactions of cyclic diazodicarbonyl compounds," Tetrahedron,vol. 58, pp. 2359-2367, 2002.

33. (a) Q. F. Wang, H. Hou, L. Hui, C. G. Yan, "Diastereoselective Synthesis of trans-2,3-Dihydrofurans with PyridiniumYlide Assisted Tandem Reaction," J. Org. Chem, vol. 74, pp. 7403-7406, 2009. (b) Y. Han, H. Hou, R. 
Yao, Q. Fu and C. G. Yan, "Diastereoselective Synthesis of trans-2, 3, 6, 7-Tetrahydro-4 (5H)-benzofuranones and trans-2, 3-Dihydrofurocoumarins via Pyridinium Ylide Assisted Tandem Reaction," Synthesis, vol. 23, pp. 4061-4067, 2010.

34. M. Yilmaz, M. Yakut and A. T. Pekel, "Synthesis of 2,3-Dihydro-4H-furo[3,2-c] chromen-4H-ones and 2,3Dihydronaphtho[2,3-b]furan-4,9-diones by the Radical Cyclizations of Hydroxyenones with Electron-Rich Alkenes using Manganese(III) Acetate,"Synth. Commun, vol. 38, pp. 914-927, 2008.

35. L. A. Shastri, M. V. Kulkarni, V. K. Gupta and N. Sharma, "Thermal Chemoselective Synthesis of Novel 2' $3^{\prime}$-Dihydro-3' -Hydroxy-benzofuranylcoumarins," Syn. Commun, vol. 38, pp. 1407-1415, 2008.

36. N. B. Yaragatti, I. N. Shcherbakov and M. V. Kulkarni, "Synthesis, modeling and biological studies on 4-2'(2,3dihydrobenzofuranyl)coumarins," ARKIVOC, vol. viii, pp. 1-16, 2012.

37. M. V. Kulkarni and V. D. Patil, "Studies on Coumarins, I," Arch. Pharm. I, vol. 314, pp. 708-711,1981.

38. A. Burger and G. E. Ullyot, "Analgesic Studies. $\beta$-ethyl and $\beta$-isopropylamine derivatives of pyridine and thiazole," J. Org. Chem, vol. 12, pp. 342-355,1947. 